Journal of Liquid Chromatography \& Related Technologies ${ }^{\circledR}$, 29: 185-201, 2006

Copyright (C) Taylor \& Francis Group, LLC

ISSN 1082-6076 print/1520-572X online

DOI: $10.1080 / 10826070500416429$

\title{
Use of an Evaporative Light Scattering Detector Coupled to MALLS for Determination of Polysaccharide Molecular Weights
}

\author{
Gary A. Luzio \\ USDA, ARS, Citrus \& Subtropical Products Research Laboratory, \\ Winter Haven, Florida
}

\begin{abstract}
A method to calibrate and use an evaporative light scattering detector (ELS) as a mass detector for molecular weight determination of polysaccharides using a multi-angle laser light scattering detector (MALLS) is described. The calibration of the ELS is performed under isocratic conditions using concentration values obtained from an interferometric refractive index detector (RI). The observed response fit a power trendline $\left(\mathrm{y}=\boldsymbol{a} \mathrm{x}^{b}\right)$ for the concentration response of the RI versus the voltage response of the ELS. The combined average $\log a$ coefficient was (5.472, s.d. $=0.020)$ and the average $\boldsymbol{b}$ coefficient was $(1.372$, s.d. $=0.004)$ for all the runs. Band broadening, which could occur between the detectors, was not observed and did not affect the calibration values. Without adjustment the ELS was used as a mass detector for MALLS to accurately determine molecular weights (MW) at elevated buffer concentrations.
\end{abstract}

Keywords: Evaporative light scattering, Multi-angle light scattering

Mention of a trademark or proprietary product is for identification only and does not imply a guarantee or warranty of the product by the US Department of Agriculture. The U.S. Department of Agriculture prohibits discrimination in all its programs and activities on the basis of race, color, national origin, gender, religion, age, disability, political beliefs, sexual orientation and marital or family status.

Address correspondence to Gary A. Luzio, USDA, ARS, Citrus \& Subtropical Products Research Laboratory, P.O. Box 1909, Winter Haven, FL 33883-1909. E-mail: gluzio@citrus.usda.gov 


\section{INTRODUCTION}

The determination of molecular weights of large molecules greater than $1 \mathrm{kD}$ is typically performed using size exclusion chromatography (SEC) columns coupled to MALLS under isocratic conditions. ${ }^{[1,2]}$ This technique requires the use of a mass detector, such as an interferometric refractometer detector (RI), to calculate the absolute molecular weight. The RI is a bulk property detector in that it measures a property of the mobile phase. Thus, it is sensitive to all changes in the mobile phase, such as increasing salt concentrations. For this reason, the RI works well under isocratic conditions, but is problematic to use when gradients are required. ${ }^{[3,4]}$

For molecular weight determinations, a MALLS is less sensitive to gradients than RI, since salt or buffer gradients involve the use of small molecules $(<1 \mathrm{kD})$ and small molecules scatter visible light waves to a much smaller extent than the large molecules typically being studied. Thus, a main limitation in doing molecular weight determinations under non-isocratic conditions with MALLS, is due to the difficulty in using the RI under these conditions as the mass detector. Other mass detectors do exist which can be used with gradients, such as a UV detector, but their use requires the presence of chromophores in the molecule being studied. With many polymers, such as most polysaccharides, there is no chromophore present to be detected by UV absorbance. ELS could also be used under non-isocratic conditions to study polysaccharides (in particular anionic or cationic polysaccharides) with MALLS using ion chromatography, but work in this area has been limited. It has been noted that there are serious problems in quantification of the ELS signal. ${ }^{[2,5,6]}$ The non-linear signal response of ELS is sensitive to a variety of parameters, which include changes in gas flow rate, solute properties, photomultiplier sensitivity, intensity of incident light, sample load, et cetera. ${ }^{[5]}$ Perhaps this has limited its use in applications involving MALLS with gradients.

The use of salt gradients has been of particular value in studying the fractionation of polysaccharides with ionic functional groups according to charge characteristics, ${ }^{[7,8]}$ and in this application RI is not used as a mass detector. In future work, it may be of value to obtain absolute molecular size data while applying a gradient elution for the study of charged polysaccharides with MALLS. The purpose of this paper is to investigate the use of ELS as a mass detector coupled with MALLS for accurately determining the molecular weights of both neutral and ionic polysaccharides such as pullulan, dextran, and pectins under isocratic conditions with different buffer concentrations. Ultimately, the goal will be to use the ELS as a mass detector for MALLS under non-isocratic conditions using ion chromatography to study ionic polysaccharides. 


\section{EXPERIMENTAL}

\section{Chemicals and Reagents}

Buffer for the mobile phase was ammonium formate at (no. 09735, $>99 \%$ purity, Fluka BioChemika, Steinheim, Switzerland) $100 \mathrm{mM}$ and $0.5 \mathrm{M} \mathrm{pH}$ 7 in high purity filtered deionized water. Polysaccharides tested were pullulan (P-4516, Sigma, St. Louis, MO), pectin esterified (P-9561 Sigma, St. Louis, MO), and dextran (D1537, labeled average MW 71,400 g/mol, Sigma, St. Louis, MO). Polysaccharides were dissolved at room temperature at a concentration of $0.5 \%$ in the filtered mobile phase.

\section{SEC MALLS Analysis}

The HPSEC-MALLS system consisted of a pump (Model 1000D syringe pump, ISCO, Lincoln, Nebraska) and an in line filter $(0.02 \mu \mathrm{m}$ pore size, Anodisc 25, Whatman, Maidstone, Eng.). Mobile phase was degassed under vacuum prior to loading into the syringe pump. Mobile phase flow rate was $0.60 \mathrm{~mL} / \mathrm{min}$. Samples were injected with a $56 \mu \mathrm{L}$ sample loop from an autosampler (Model 728, Alcott, Norcross, GA) through an injection valve (Model 732, Alcott, Norcross, GA) onto a set of three linear (PL-aquagel-OH 50 and $60,8 \mu \mathrm{m}$ pore size, $300 \times 5.7 \mathrm{~mm}$ from Polymer Laboratories Inc., Amherst, MA and TSK-GEL, $10 \mu \mathrm{m}$ pore size, $600 \times 7.5 \mathrm{~mm}$ from TOSOHaas, Montgomeryville, PA) SEC columns with an operating range of $100-$ $10,000,000 \mathrm{MW}$. The columns were connected in series (largest pore size first), enclosed in a column heater, and kept at $37.00 \pm .02^{\circ} \mathrm{C}$. Three detectors were present in line in the following order: MALLS, RI, and ELS. The MALLS (DAWN ${ }^{\circledR}$ EOS, Wyatt Technologies, Santa Barbara, CA) was equipped with a K5 flow cell and a $\mathrm{He}-\mathrm{Ne}$ laser light source $(\mathrm{L}=633 \mathrm{~nm})$. Prior to the measurements, the MALLS was calibrated using $0.2 \mathrm{~mm}$ filtered HPLC quality toluene. The RI (Optilab ${ }^{\circledR}$ DSP, Wyatt Technologies, Santa Barbara, CA) had a P100 cell (10 mm path length) operating at $633 \mathrm{~nm}$ and a constant temperature of $42^{\circ} \mathrm{C}$. The ELS (Model 301, ESA Inc., Chelmsford, MA) was set at a photomultiplier gain setting of $700 \mathrm{mV}$, a nebulizer setting of $45^{\circ} \mathrm{C}$, and an evaporation temperature of $150^{\circ} \mathrm{C}$. Nitrogen gas flow rate was measured at $1.95 \mathrm{~L} / \mathrm{min}$ and nitrogen gas was filtered through a gas filter (Models PF 7903-00010 and PM16-03-F0 1103, Bosch Rexroth, Hoffman Estates, IL), and regulated at 20 psi (Model PR7903-00010, Bosch Rexroth, Hoffman Estates, IL).

Conditions for calibration of the ELS with concentration data from the RI were as follows. The mobile phase was $100 \mathrm{mM}$ ammonium formate at $0.6 \mathrm{~mL} / \mathrm{min}$. Polysaccharides solutions were filtered through $0.45 \mu \mathrm{M}$ filter before injection. Data was collected at 1.25 second intervals. Results were 
processed using the software provided by the manufacturer (DNDC for Windows vs. 5.90.03, and Astra for Windows vs. 4.90.07, Wyatt Technologies). Elution data and molecular weight (as weight average) were processed or calculated using Astra software. Previously published values of $\mathrm{dn} / \mathrm{dc}$ were used for MW determination and mass recovery. ${ }^{[9,10]}$ Processed data was exported to a spreadsheet and adjusted for interdetector delay volume and baseline. The ELS was calibrated using regression analysis of log ELS voltage against log RI concentration calculated from the observed voltages from the RI. The RI concentration was computed from the voltage response of the RI, the RI calibration (Aux $=2.1533 \times 10^{-5}$ volts $^{-1}$ ) constant, and a dn/dc value of $0.148 \mathrm{~mL} \mathrm{~g}^{-1}$. Data from the MALLS was then processed using concentration values calculated from the ELS voltage response to determine MW for each volume fraction $(12.5 \mu \mathrm{L}$ slice) from the chromatogram and the average MW was computed from all volume fractions under the peak.

Conditions for chromatography at $0.5 \mathrm{M}$ ammonium formate concentration were as follows. The RI was purged before each run. No adjustments in baseline signal were made to ELS when switching from $100 \mathrm{mM}$ to $0.5 \mathrm{M}$ ammonium formate. Mobile flow rate was also $0.60 \mathrm{~mL} / \mathrm{min}$ and all other conditions were held constant. Sample data for $0.5 \mathrm{M}$ concentration was processed the same as with the calibration procedure with $100 \mathrm{mM}$ buffer, except the ELS signal was converted to concentration using the average values for $\boldsymbol{a}$ coefficient and $\boldsymbol{b}$ coefficient obtained from the isocratic runs using $100 \mathrm{mM}$ buffer.

\section{RESULTS AND DISCUSSION}

\section{Calibration of an ELS Using Concentration Values from an RI}

As noted, there may be difficult problems with the quantification of an ELS signal. Since the RI provides accurate concentration data during isocratic runs and is typically used with MALLS systems, the RI was tested to determine if it could be used to calibrate the ELS under isocratic conditions. Three different polysaccharides were injected at least three times over a 24 hour period. The ELS voltage response was plotted against the RI concentration calculated. Figure 1 shows a typical response curve for pullulan. The response curve was similar for all three polysaccharides examined. The response fits a power trendline $\left(\mathrm{y}=\boldsymbol{a} \mathrm{x}^{b}\right)$ for the concentration response of the RI versus the voltage response of the ELS, which is similar to previous results. ${ }^{[3]}$ The $\log \boldsymbol{a}$ coefficient value for this particular plot was 5.489, the $\boldsymbol{b}$ coefficient value was 1.377 , and the $\mathrm{R}^{2}$ value was 0.9996 . In addition, this plot contains the voltage response for the high molecular weight data points as well as the low molecular weight data points, and both sets fit the same power function. When the data points were analyzed separately based on 


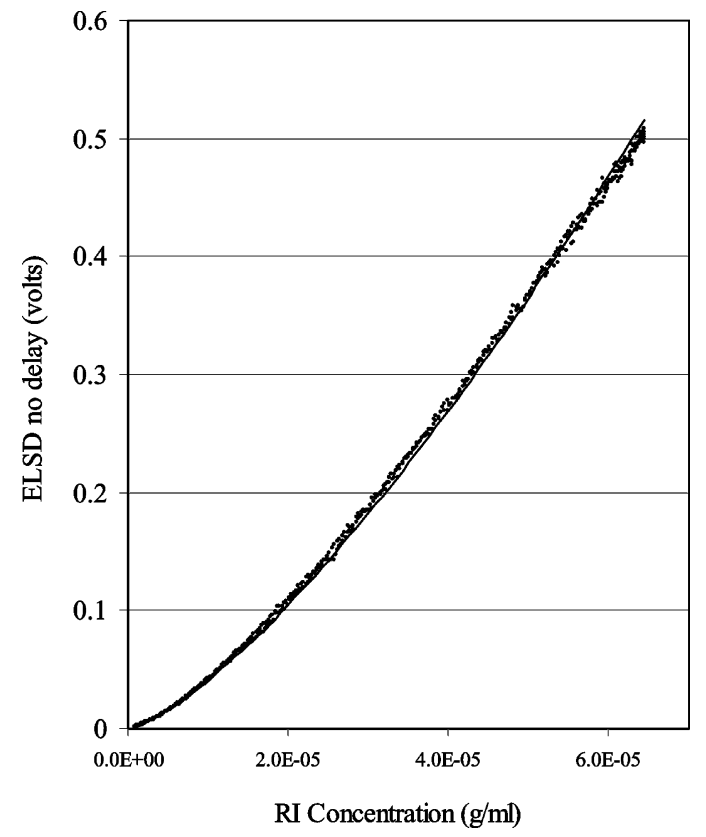

Figure 1. Voltage response of ELS versus concentration calculated from RI response for $56 \mu \mathrm{L}$ injection of $0.5 \%$ pullulan solution in $100 \mathrm{mM}$ ammonium formate. (•) data points, (-) power trendline fit to data. Concentration computed from RI Aux constant $\left(\right.$ Aux $=2.1533 \times 10^{-5}$ volts $\left.^{-1}\right), \mathrm{dn} / \mathrm{dc}$ of dextran $0.148 \mathrm{~mL} \cdot \mathrm{g}^{-1}$ and RI voltage response.

molecular weight before and after the peak maximum, the $\boldsymbol{b}$ coefficients for the high and low MW data points were 1.376 and 1.378, respectively, and the $\log \boldsymbol{a}$ coefficient values for the high and low MW data points were 5.480 and 5.494, respectively, for this particular sample with $\mathrm{R}^{2}$ values of 0.9996 for both sets of data. Similar results were observed for MW ranges from $10^{6}$ to $10^{4}$ for the three polysaccharides tested (data not shown). These data indicate that for this range of MW the ELS response is independent of MW. This is consistent with previous work, which involved high molar mass oligomers. ${ }^{[4]}$ The power trendline extended to low ELS voltages (or low concentrations) data not shown. Again, low MW values and high MW values followed the same power trendline.

It has been noted previously, that correctly setting the interdetector delay volume is important for proper quantification ${ }^{[11,12]}$ and that alignment typically should be done to approximately the nearest second. ${ }^{[12]}$ Manual alignment of the signal responses is feasible here since both detectors are sensitive to changes in concentration and are insensitive to changes in molecular weight. Other alignment procedures, besides manual alignment, for interdetector delay may be necessary if the detectors are sensitive to 
properties, which are different for each detector. ${ }^{[12]}$ Data was collected at 1.25 second intervals for all runs, which at this flow rate represents an elution volume fraction of $12.5 \mu \mathrm{L}$. Figure 2 shows the response if the interdetector delay is purposely offset by $50 \mu \mathrm{L}$ ( 5 second error) from its correct setting between the RI and the ELS. This generates a curve which simulates a type of hysteresis loop whereby voltages on both sides the elution peak are incorrectly assigned their respective concentrations. Thus, all high MW species are on one side of the fitted line and all low MW species fall on the other side. Proper alignment was checked for each separate run using the spreadsheet before log calibration was performed. In most instances, no manual shift was required and in a few instances a shift of $12.5 \mu \mathrm{L}$ delay volume (or 1.25 seconds shift in delay time) was needed to eliminate a slight hysteresis loop. Regardless, it was observed that an error of only 1.25 seconds in delay did not alter $\boldsymbol{a}$ and $\boldsymbol{b}$ coefficients from the resulting standard curve. It is difficult to calculate an absolute delay volume for ELS, since the calculation would involve a time of particle flight from the nebulizer to the detector chamber. Small changes, between runs, in gas flow rate (or perhaps liquid flow which could change nebulization) could alter the interdetector delay

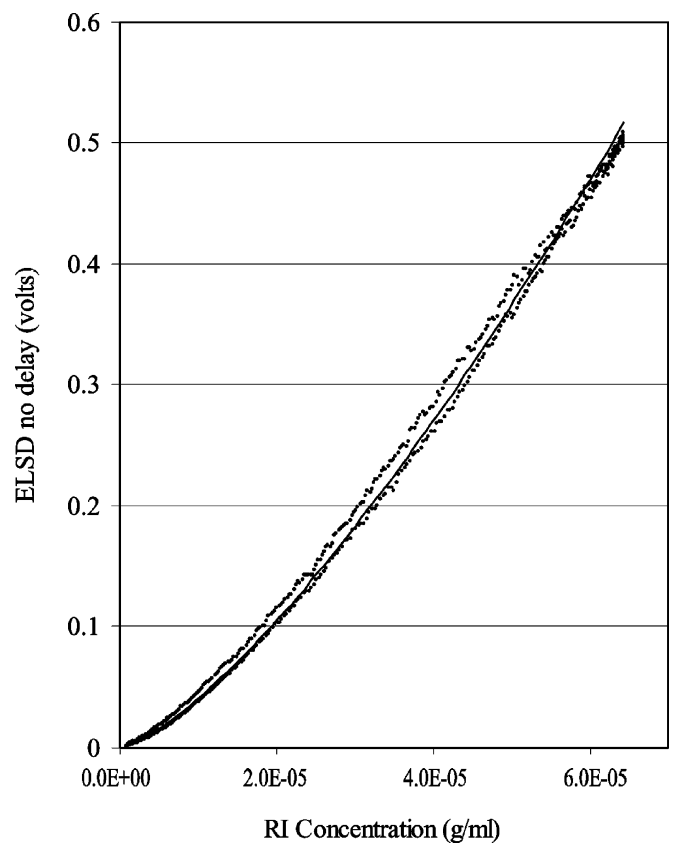

Figure 2. Inter-detector delay volume intentionally off set $50 \mu \mathrm{L}$ (equivalent to 5 second error in elution time) from correct setting with voltage response of ELS versus concentration data points calculated from RI for $56 \mu \mathrm{L}$ injection of $0.5 \%$ dextran solution in $100 \mathrm{mM}$ ammonium formate. (•) data points, (-) power trendline fit to data. 
volume. Thus, alignment was checked following each calibration run, but typically adjustments were unnecessary.

With proper setting of the baselines and setting of the interdetector delay volume the data was plotted as the log of the ELS voltage against the log of the RI concentration. A representative log plot is shown in Figure 3 for a sample of pullulan. This particular plot contains 663 calibration points, which is typical of the number of data points collected for a calibration curve. For a power function where $\mathrm{y}=\boldsymbol{a} \mathrm{x}^{b}$, the slope from the regression fit gives the $\boldsymbol{b}$ coefficient and the antilog of the intercept gives the $\boldsymbol{a}$ coefficient. The $\boldsymbol{b}$ coefficient for this calibration curve was 1.376 , the $\log \boldsymbol{a}$ coefficient was 5.478 and the $\mathrm{R}^{2}$ value was 0.9992 . Similar log plot slopes, intercepts, and $\mathrm{R}^{2}$ values were observed for pectin and pullulan calibration curves.

Several chromatograms were collected for each polysaccharide using $100 \mathrm{mM} \mathrm{NH}_{4}$ formate as the mobile phase. No changes were made in the instrument settings between runs and calibration curves were generated for each run. As shown in Table 1, the respective average $\log \boldsymbol{a}$ coefficient for pectin, dextran, and pullulan was 5.630, 5.543, and 5.601 with s.d. values of 0.05 or less. The respective average $\boldsymbol{b}$ coefficient for pectin, dextran, and pullulan was $1.406,1.391$, and 1.397 with s.d. values of 0.008 or less. With

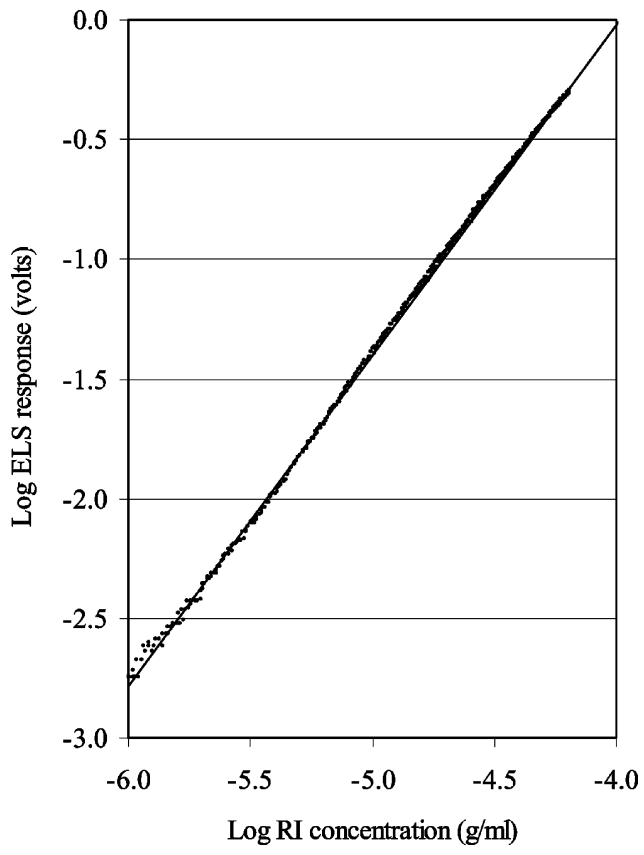

Figure 3. Log voltage response of ELS versus log concentration calculated from RI for $56 \mu \mathrm{L}$ injection of $0.5 \%$ pullulan solution in $100 \mathrm{mM}$ ammonium formate. ( $\bullet$ ) data points, (-) linear trendline fit to data. 


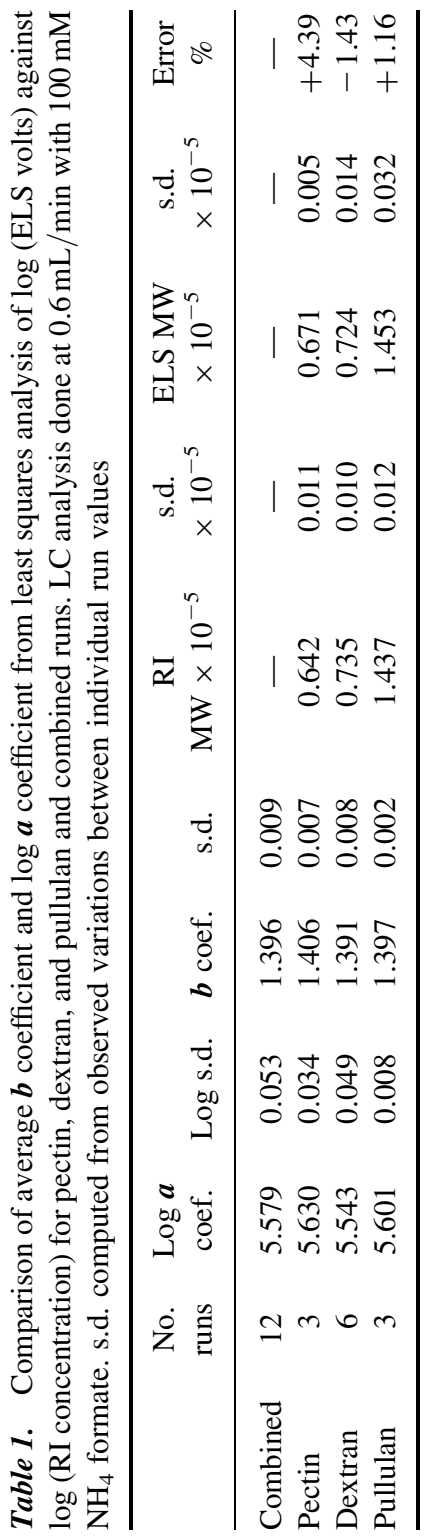


both the $\log$ ( $\boldsymbol{a}$ coefficient) and $\boldsymbol{b}$ coefficient, the values were within one s.d. of the average for the combined runs regardless of the polysaccharide tested. These data indicate that the calibration curve is independent of the type of polysaccharide used for calibration. This is consistent with previous findings that indicate for compounds with similar refractive indexes and densities, the expected response from the ELS is the same for both assuming there are no chromophores in one of the compounds, which could absorb the light scattering signal. ${ }^{[3]}$

All runs were then averaged to obtain a combined value. The combined average $\log \boldsymbol{a}$ coefficient was $(5.579$, s.d. $=0.053)$ and the average $\boldsymbol{b}$ coefficient was $(1.396$, s.d. $=0.009)$ for all the runs is given in Table 1 . The average correlation coefficient (values not in table) for the log calibration curves for the 12 runs was 0.9985 with an s.d. of 0.0014 . The low s.d. value for each of the polysaccharide runs and for the averages of all values obtained indicated that the calibration curve is reproducible, and would be useful for determining the concentration or mass of a polysaccharide for use in a molecular weight determination by MALLS.

The combined average $\boldsymbol{a}$ coefficient and the average $\boldsymbol{b}$ coefficient were used in the calculation of average MW for each of the three polysaccharides from the ELS voltage response and the data from the MALLS. Figure 4 shows a typical chromatogram of the signals from each detector overlaid with MWs from the RI and the ELS as computed from combined average value of all the $\boldsymbol{a}$ and $\boldsymbol{b}$ coefficients. For this particular run, using pullulan, the average MW from the RI was $1.47 \times 10^{5}$ and from the ELS was $1.46 \times 10^{5}$. The average MWs of all runs are shown in Figure 5, with the actual values given in Table I. For example with dextran, the average MW was $0.735 \times 10^{5}$ with an s.d. of $0.01 \times 10^{5}$ for the RI, and for the ELS the average MW calculated was $0.724 \times 10^{5}$ with an s.d. of $0.014 \times 10^{5}$. The error for the ELS was $-1.43 \%$ as compared to the RI for dextran. Similar results were obtained for pectin and pullulan. These data indicate that the ELS can be calibrated and used as an accurate mass detector for MALLS under isocratic conditions, independent of the type of polysaccharide used for calibration.

\section{Effect of Potential Band Broadening on Calibration of ELS}

Aside from incorrectly setting the interdetector delay volume or baselines for each detector, another potential error is peak band broadening, which could occur in the connecting tubing by diffusion as the sample flows from the RI to the ELS or dispersion in the volume of the RI detector cell. ${ }^{[13,14]}$ If band broadening is significant during the transfer from one detector to another or by sample flow through a detector cell, then the concentration observed by the ELS at any given point is not the same as the RI, and calibration would not be feasible by this technique. Band broadening is a significant problem 


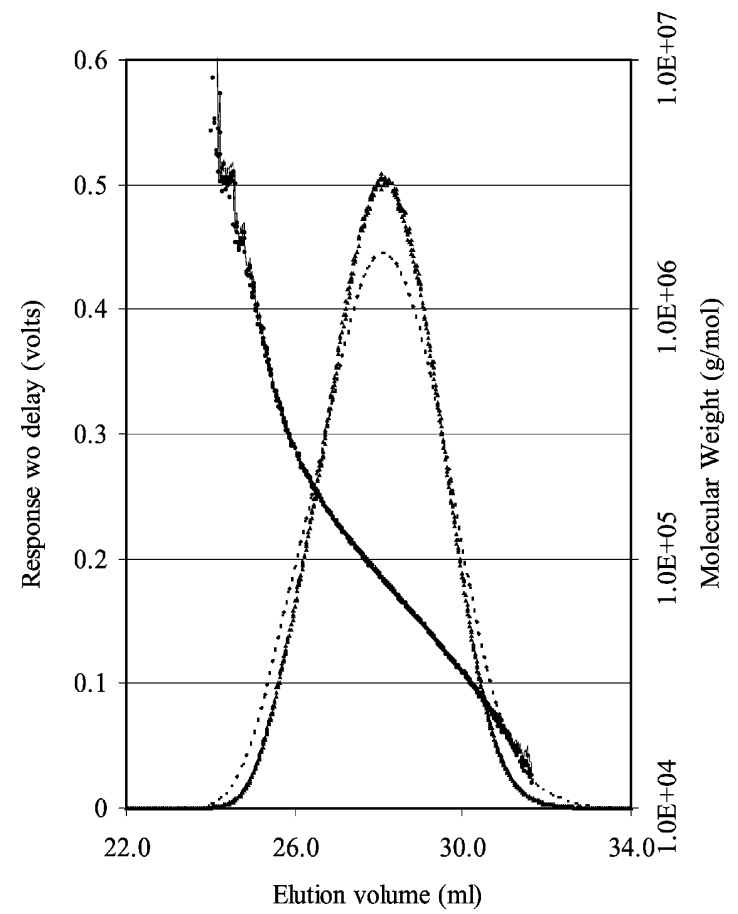

Figure 4. Chromatogram of RI voltage response (- - - -) in $100 \mathrm{mM}$ ammonium formate for $56 \mu \mathrm{L}$ injection of $0.5 \%$ pullulan solution versus ELS voltage response ( $\mathbf{\Delta})$ overlaid with MW calculated from RI response (-) and MW from ELS response $(\bullet)$ ). MW for RI computed from RI Aux constant $\left(\right.$ Aux $\left.=2.1533 \times 10^{-5}\right), \mathrm{dn} / \mathrm{dc}$ of $0.148 \mathrm{~mL} \mathrm{~g}^{-1}$ and RI voltage. ELS response computed from $\mathrm{x}=(\mathrm{y} / \mathrm{a})^{1 / \mathrm{b}}$ where $\mathrm{y}$ is the voltage response from ELS, $\boldsymbol{b}=1.396$ and $\boldsymbol{a}=1 \times 10^{5.579}$.

with narrow eluting peaks. ${ }^{[15]}$ In contrast, for broad chromatograms involving polydisperse systems, the concentration values are relatively unchanged except for perhaps the extreme tails of the chromatograms. ${ }^{[16]}$ The peaks being used here for calibration are broad and the use of polydisperse samples is intentional since it could minimize the effects of band broadening and also provides a wide range of concentration values for calibration. Computations could be performed to estimate the potential for band broadening by transfer of the sample through the tubing, ${ }^{[13]}$ but the most straightforward approach is to perform direct measurements using the ELS with and without the RI in line, to determine if band broadening is affecting the calibration.

In the experiment, the RI was removed and all three polysaccharides were retested in triplicate to examine effects on peak shape and MW values as compared to the data with the RI in line. In Figure 6, the chromatogram of the voltage response from the ELS with the RI in line is overlaid with the chromatogram of the voltage response from the ELS with no RI in line. The sample 


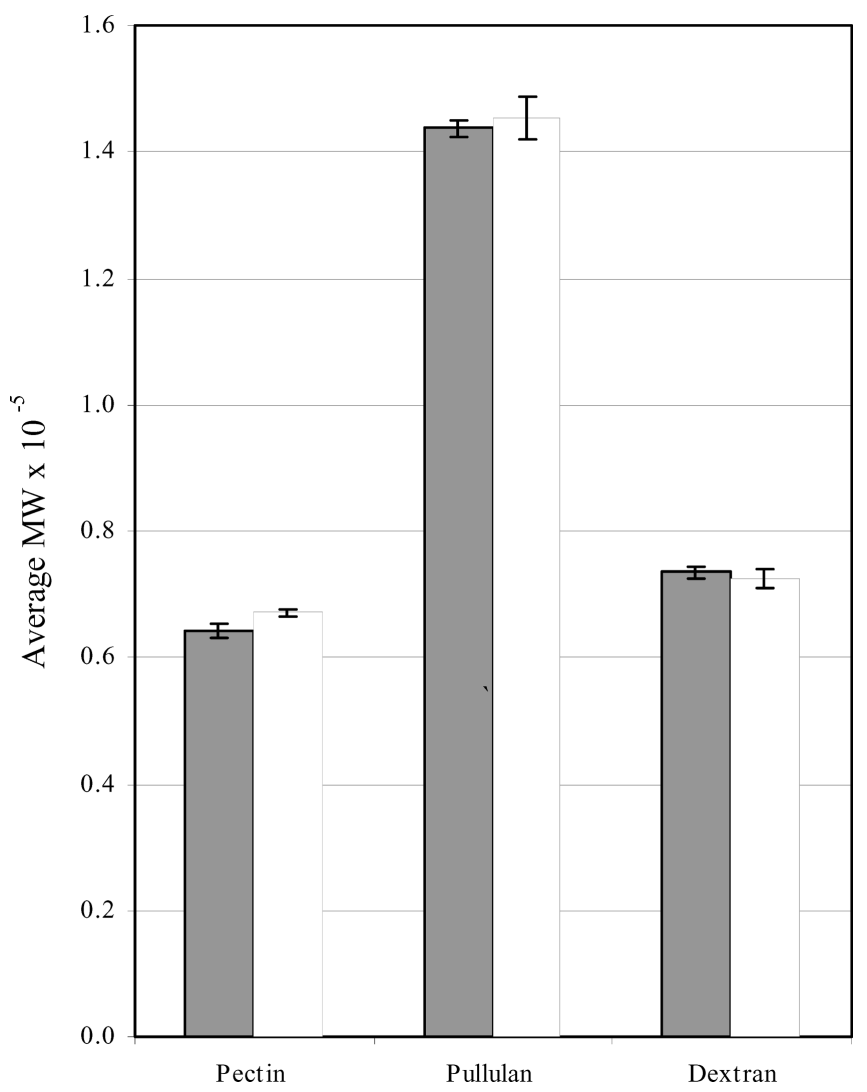

Figure 5. Average MW for samples of pectin, pullulan and dextran calculated from RI response ( $\square$ ) using RI voltages, Aux constants and dn/dc values noted previously and calculated from ELS response $(\square)$ using average coefficients $(\log a=5.579$ and $\boldsymbol{b}=1.396)$ computed from 12 calibration injections using all three polysaccharides with $100 \mathrm{mM}$ ammonium formate mobile phase.

used was pullulan. In addition the MW values for the two separate experiments are also overlaid. The solid line represents the MW values and the voltage response obtained from the ELS with no RI in line. The solid line is difficult to observe on the plot since it is overlaid with the data points from the ELS with the RI in line. This would be expected if band broadening is not a significant factor with the wide peaks which are eluting. As shown by visual inspection, there is no effect on peak shape, peak height, peak width at baseline, and peak width at half peak height, by inclusion of the RI in line. Even at the tails of the chromatogram there was no observed difference in response of the ELS. These data indicate that peak broadening, which may occur between the RI and the ELS did not affect the calibration of the ELS. Similar results were observed with all three polysaccharides tested. 


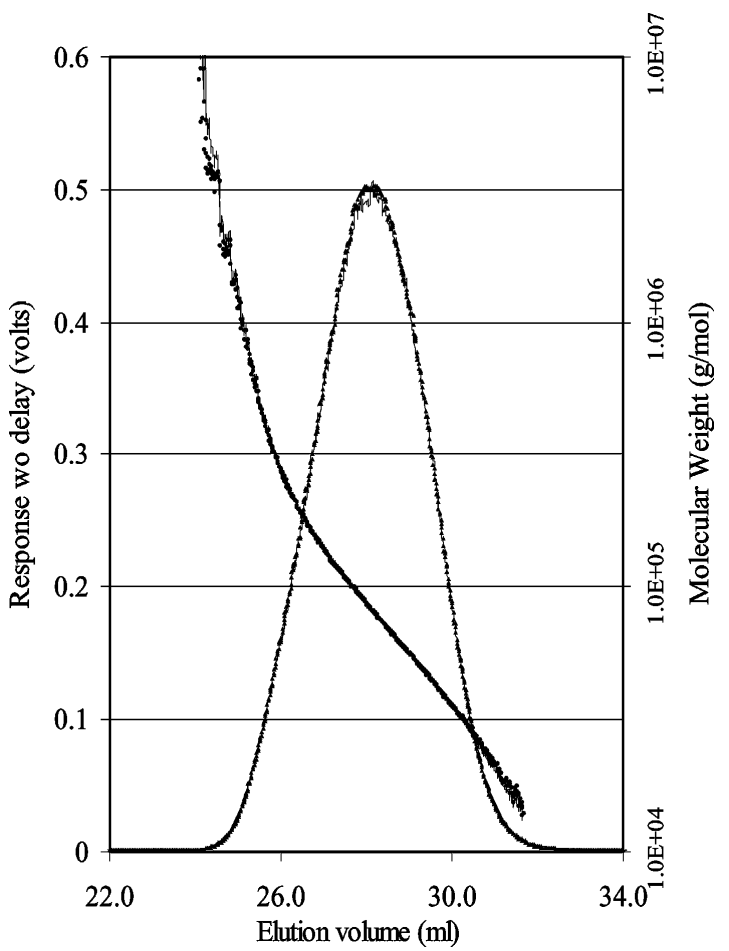

Figure 6. Chromatogram of ELS voltage response with no RI in line (-) and ELS response with RI in line $(\mathbf{\Lambda})$ overlaid with MW obtained from ELS response with no RI in line (-) and MW from ELS response with RI in line $(\bullet)$ for $56 \mu \mathrm{L}$ injection of $0.5 \%$ dextran solution in $0.5 \mathrm{M}$ ammonium formate as computed from $\mathrm{x}=(\mathrm{y} / \mathrm{a})^{1 / \mathrm{b}}$ where $y$ is the voltage response from ELS, $\boldsymbol{b}=1.396$ and $\boldsymbol{a}=1 \times 10^{5.579}$. Data plotted against elution volume with adjustments made for delay volume.

To further illustrate this, the voltage response (without delay) from the ELS without the RI in line was plotted against the voltage response (without delay) from the ELS with the RI in line, as shown in Figure 7 using a sample of pullulan. As shown, there are no significant deviations from the linear trendline and the slope of the line is 0.992 with and $\mathrm{R}^{2}$ value of 0.9996 . This would be expected if band broadening is not a significant factor, as the sample is transferred through the tubing from the RI to the ELS.

In addition, visual observation of the MW curve shows no significant deviation with and without the RI in line as shown in Figure 6. To further establish that peak broadening does not affect the MW results, the average MWs were calculated with and without the RI in line and the results are given in Table 2. The maximum error observed was with pullulan, where the average MW obtained was $1.38 \%$ higher, when the RI was in line as compared to the MW when the RI was removed from the system. These 


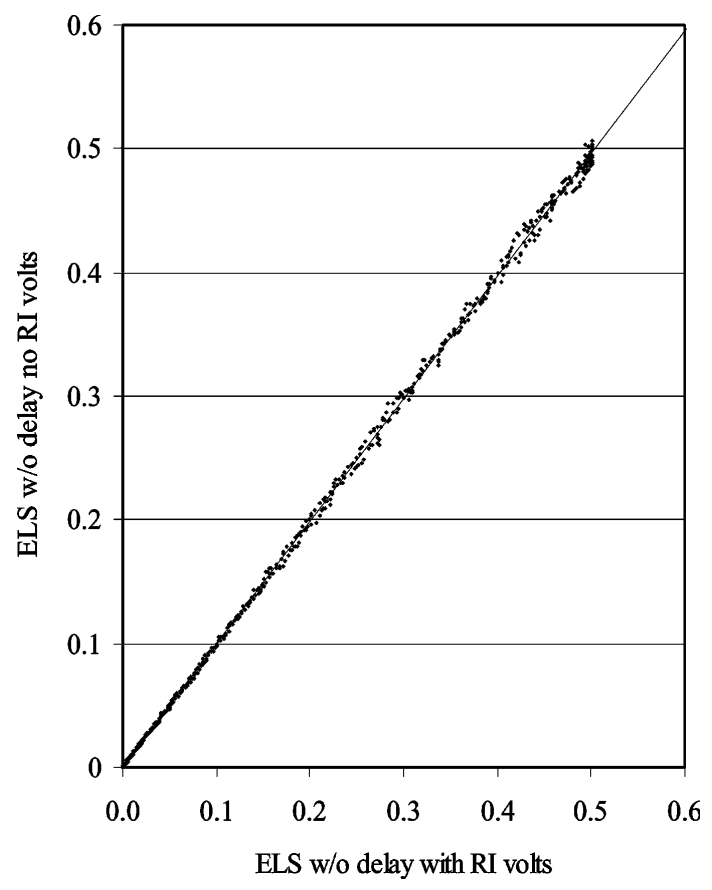

Figure 7. ELS voltage response with no RI in line versus ELS response with RI in line for $56 \mu \mathrm{L}$ injection of $0.5 \%$ dextran solution in $0.5 \mathrm{M}$ ammonium formate with data adjusted for delay volume. $(\bullet)$ data points, $(-)$ linear trendline.

data indicate that band broadening, which could occur when the sample is transferred from one mass detector to the other, does not affect the results using these polydisperse samples. It should be noted that this calibration is valid for broadly eluting peaks. For monodisperse polymers or narrow eluting peaks, proper quantitation (after calibration) would require the removal of the RI, since band broadening could be significant. Monodisperse or narrowly eluting polymers probably could not be properly quantified with the RI in line.

\section{Determination of Molecular Weight at Elevated Buffer Concentrations}

To determine what effect buffer concentration has on the calibration curve, the three polysaccharide samples were chromatographed at an elevated buffer level. The mobile phase was $0.5 \mathrm{M} \mathrm{NH}_{4}$ formate and runs were performed in triplicate. No adjustment was made to the baseline signal of the ELS when switching from $100 \mathrm{mM}$ to the $0.5 \mathrm{M}$ ammonium formate. The molecular weight was calculated from the ELS and MALLS signals using 


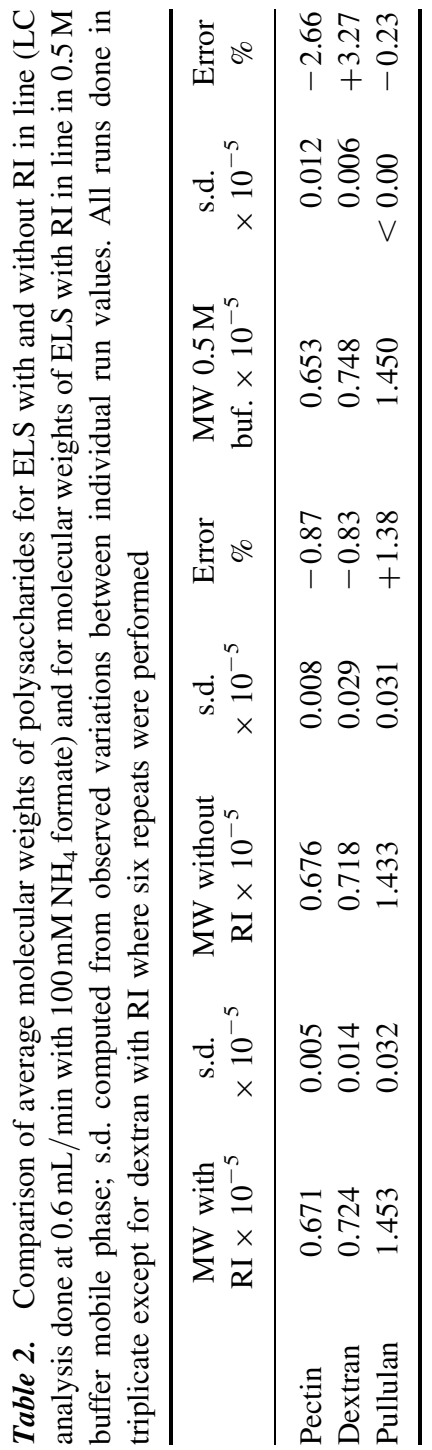




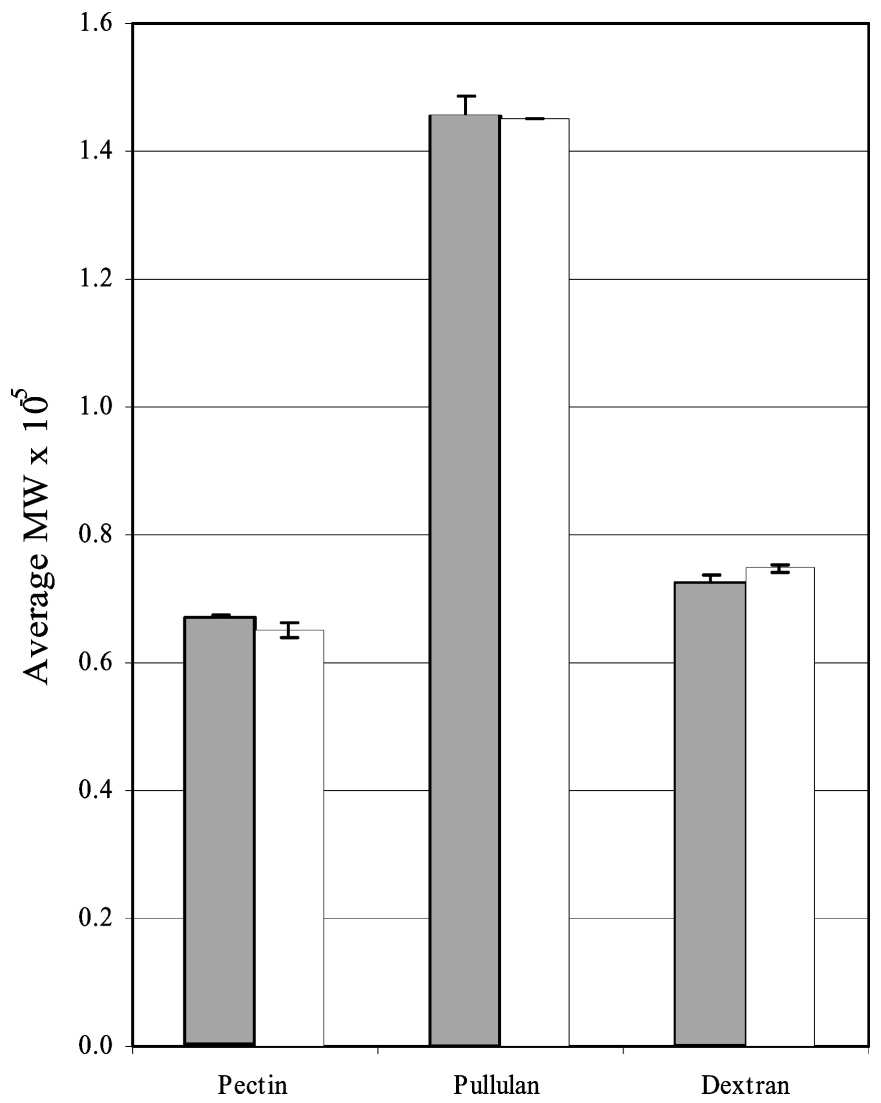

Figure 8. Average MW for pectin, pullulan and dextran ELS using $0.1 \mathrm{M}$ ammonium formate buffer mobile phase and $\square$ from ELS using $0.5 \mathrm{M}$ ammonium formate buffer. All data computed using average coefficient $(\log \boldsymbol{a}=5.579$ and $\boldsymbol{b}$ (1.396) coefficient from all 12 calibration injections performed $100 \mathrm{mM} \mathrm{NH}_{4}$ ammonium formate mobile phase with RI providing concentration values.

the average $\boldsymbol{a}$ coefficient and $\boldsymbol{b}$ coefficient obtained from the standard curves at $100 \mathrm{mM}$ ammonium formate. A comparison of average MWs for all three polysaccharides obtained from the ELS for the $100 \mathrm{mM}$ and $500 \mathrm{mM}$ ammonium formate is shown in Figure 8. For example, the average MW for dextran from the ELS (in $0.500 \mathrm{M}$ ammonium formate) was $0.748 \times 10^{5} \mathrm{~g} / \mathrm{mol}$ with an s.d. of $0.006 \times 10^{5}$ and in $100 \mathrm{mM}$ ammonium formate the average MW was $0.724 \times 10^{5} \mathrm{~g} / \mathrm{mol}$ with an s.d. of $0.014 \times 10^{5}$. Similar data was observed for the other two polysaccharides (results in Table 2). These data indicate that under these ELS settings ammonium formate concentration does not have a significant effect on the mass values and, thus, the MW values obtained. These data indicate that, after calibration, the ELS might be useful with ion chromatography to 
determine mass values for polysaccharides for the determination of MW for the MALLS while performing gradient elution.

\section{CONCLUSION}

It has been shown that the concentration values obtained from an RI is effective for the calibration of ELS for quantification of polysaccharides using polydisperse polysaccharides for calibration. Band broadening, which is a potential error in calibration, is not significant when using these polydisperse samples. The ELS could then be used to determine the mass value in the molecular weight determination by MALLS in isocratic runs, and also gradient experiments, after switching the system to ion chromatography following calibration under isocratic conditions.

\section{REFERENCES}

1. Wyatt, P.J. Light scattering and the absolute characterization of macromolecules. Anal. Chim. Acta 1993, 272, 1-40.

2. Trathnigg, B. Size-exclusion chromatography of polymers. In Encyclopedia of Analytical Chemistry; 2000; 8008-8034.

3. Charlesworth, J.M. Evaporative analyzer as a mass detector for liquid chromatography. Anal. Chem. 1978, 50, 1414-1420.

4. Trathnigg, B.; Kollroser, M. Liquid-chromatography of polyethers using universal detectors. Quantitative aspects in the analysis of low-molecular mass polyethylene glycols and their derivatives by reversed-phase high-performance liquidchromatography with an evaporative light-scattering detector. J. Chromatogr. A 1997, 768, 223-238.

5. Mourey, T.H.; Oppenheimer, L.E. Principles of operation of an evaporative light scattering detectors for liquid chromatography. Anal. Chem. 1984, 56, 2427-2434.

6. Van der Meeren, P.; Vanderdeelan, J.; Baert, L. Simulation of the mass response of the evaporative light scattering detector. Anal. Chem. 1992, 64, 1056-1062.

7. Cameron, R.G.; Hotchkiss, A.T.; Kauffman, S.W.; Grohmann, K. Utilization of an evaporative light scattering detector for high performance size exclusion chromatography of galacturonic acid oligomers. J. Chromatogr. A 2003, 1011, 227-231.

8. Ralet, M.-C.; Bonnin, E.; Thibault, J.-F. Chromatographic study of highly methoxylated lime pectins deesterified by different methyl-esterases. J. Chromatogr. B 2001, 753, 157-166.

9. Fishman, M.L.; Chau, H.K.; Hoagland, P.; Ayyad, K. Characterization of pectin flash-extracted from orange albedo by microwave heating under pressure. Carb. Res. 2000, 323, 126-138.

10. Nordmeier, E. Static and dynamic light-scattering solution behavior of pullulan and dextran in comparison. J. Phys. Chem. 1993, 97, 5770-5785.

11. Mourey, T.H.; Balke, S.T. A strategy for interpreting multidetector size-exclusion chromatography data. I. development of a systematic approach. ACS Symp. Ser. 1993, 521, 180-198. 
12. Zammit, M.D.; Davis, T.P.; Suddaby, K.G. Factors influencing detector matching in multidetector SEC: solvent and concentration effects. Polymer 1998, 39, 5789-5798.

13. Scott, R.P. Extra-column dispersion in liquid chromatography systems. J. Liq. Chromatogr. \& Rel. Technol. 2002, 17, 2567-2587.

14. Netopilik, M. Influence of peak-broadening and interdetector volume error on sizeexclusion chromatographic analysis with dual viscometric-concentration detection using the universal calibration method. J. Chromatogr. A 2001, 915, 15-24.

15. Suddaby, K.G.; Sanayei, R.A.; Driscoll, K.F.; Rudin, A. Multi-detector size exclusion chromatography: a novel approach to calibration eliminating lag time estimation. Makromol. Chem. 1993, 194, 1965-1974.

16. Cheung, P.; Lew, R.; Balke, S.T.; Mourey, T.H. SEC-viscometer detector systems. II. resolution correction and determination of interdetector volume. J. Appl. Poly. Sci. 1993, 47, 1701-1706.

Received August 20, 2005

Accepted September 22, 2005

Manuscript 6698 
